ARTICLE

DOI: $10.1038 /$ s41467-017-00656-8 OPEN

\title{
In situ wrapping of the cathode material in lithium- sulfur batteries
}

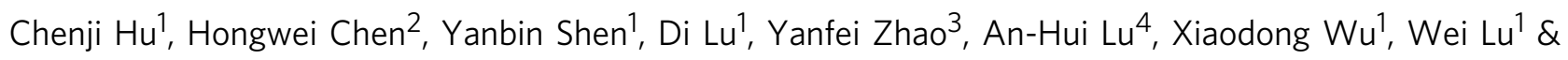
Liwei Chen (1) 1,3

While lithium-sulfur batteries are poised to be the next-generation high-density energy storage devices, the intrinsic polysulfide shuttle has limited their practical applications. Many recent investigations have focused on the development of methods to wrap the sulfur material with a diffusion barrier layer. However, there is a trade-off between a perfect preassembled wrapping layer and electrolyte infiltration into the wrapped sulfur cathode. Here, we demonstrate an in situ wrapping approach to construct a compact layer on carbon/ sulfur composite particles with an imperfect wrapping layer. This special configuration suppresses the shuttle effect while allowing polysulfide diffusion within the interior of the wrapped composite particles. As a result, the wrapped cathode for lithium-sulfur batteries greatly improves the Coulombic efficiency and cycle life. Importantly, the capacity decay of the cell at 1000 cycles is as small as $0.03 \%$ per cycle at $1672 \mathrm{~mA} \mathrm{~g}^{-1}$.

\footnotetext{
${ }^{1}$ i-Lab, CAS Center for Excellence in Nanoscience, Suzhou Institute of Nano-Tech and Nano-Bionics (SINANO), Chinese Academy of Sciences, Suzhou 215123, China. ${ }^{2}$ College of Materials Science and Engineering, Huaqiao University, Xiamen 361021, China. ${ }^{3}$ Vacuum Interconnected Nanotech Workstation, SINANO, Chinese Academy of Sciences, Suzhou 215123, China. ${ }^{4}$ State Key Laboratory of Fine Chemicals, School of Chemical Engineering, Dalian University of Technology, Dalian 116024, China. Chenji Hu and Hongwei Chen contributed equally to this work. Correspondence and requests for materials should be addressed to H.C. (email: hwchen@hqu.edu.cn) or to L.C. (email: Iwchen2008@sinano.ac.cn)
} 
R echargeable lithium-sulfur (Li-S) batteries are widely expected to be the next-generation high-density energy storage technology since the low-cost sulfur cathode has a high theoretical specific capacity of $1672 \mathrm{mAhg}^{-11,2}$. The operating mechanism of a Li-S battery is based on a series of redox reactions between elemental sulfur $\left(\mathrm{S}_{8}\right)$ and its polysulfide derivatives $\mathrm{Li}_{2} \mathrm{~S}_{n}{ }^{2-}(1 \leq n \leq 8)$. The cathode material experiences a complex phase transition from solid $\mathrm{S}_{8}$ to dissolved polysulfide ions to insoluble $\mathrm{Li}_{2} \mathrm{~S}_{2}$ and $\mathrm{Li}_{2} \mathrm{~S}$. When combined with the reversible lithium metal stripping/plating process at the anode, the Li-S batteries provide a theoretical energy density of 2600 $\mathrm{Wh} / \mathrm{kg}$, which assumes the complete conversion of $\mathrm{S}_{8}$ to $\mathrm{Li}_{2} \mathrm{~S}^{3}$.

However, the commercialization of Li-S technology depends on the solution to multiple critical issues that involve the electrolyte and Li anode. For example, one of the key problems in Li-S batteries has been the shuttle effect in the cathode that is described as follows. First, the polysulfide ions that are formed at the cathode are dissolved in the electrolyte and may diffuse to the anode where they are reduced to lower polysulfides. Then, the ions diffuse back to the cathode where they are reoxidized. This back and forth transport, i.e., "shuttle" between the cathode and anode may be continuous ${ }^{4}$ and thus give rise to the deposition of non-conductive $\mathrm{Li}_{2} \mathrm{~S}_{2}$ or $\mathrm{Li}_{2} \mathrm{~S}$ on the $\mathrm{Li}$ anode, the consumption of sulfur species and a poorly controlled $\mathrm{Li} /$ electrolyte interface. These effects lead to a low Coulombic efficiency, a high self-discharging rate and a fast decay in capacity (Fig. 1a, the "no wrapping" case) $)^{5}$.

Various approaches have been devised to suppress the shuttle effect $^{6-14}$, among which a major strategy is to wrap the sulfur material with a diffusion barrier layer to confine the solvated polysulfides within the cathode material. The sulfur wrapping strategy typically involves the preparation of carbon-sulfur composite particles with a wrapping layer and then the wrapped $\mathrm{C} / \mathrm{S}$ composite is assembled into coin cells for testing. Such a pre-battery-assembly wrapping approach has achieved good results, which include a greatly suppressed shuttle effect and improved cycling stability ${ }^{5-17}$. However, a dilemma exists in designing the wrapping layer when using this approach, as illustrated in Fig. 1b. If the preassembly wrapping layer was designed to be perfectly compact and tight (to completely block the polysulfide diffusion), the electrolyte would not infiltrate the $\mathrm{C} / \mathrm{S}$ composite in the assembled cell. Then, the battery will exhibit poor performance. Alternatively, if the preassembly wrapping layer was imperfectly designed with pores and/or cracks that allow for the penetration of the electrolyte into the $\mathrm{C} / \mathrm{S}$ composite, solvated polysulfides can also leak out of the wrapping layer via these defects, which can lead to improved but diminishing performance (Fig. 1c). The latter scenario represents most sulfur wrapping work reported to date ${ }^{15-17}$.

Here, for the first time, we propose and demonstrate an in situ wrapping approach. In Fig. 1d, C/S composite particles are imperfectly coated with a wrapping layer, and the material is assembled into coin cells. The imperfect wrapping layer allows the infiltration of adequate electrolyte into the $\mathrm{C} / \mathrm{S}$ composite particles. Significantly, a special functional additive is added to the electrolyte to react with the initially imperfect wrapping layer to form a second wrapping layer after the battery is assembled. The second, post-assembled and in situ-formed wrapping layer is designed to be compact and tight to completely block the shuttle effect, which allows polysulfide dissolution into the electrolyte within the interior of the wrapped composite particles. The coin cells with an in situ wrapped C/S cathode demonstrate an initial

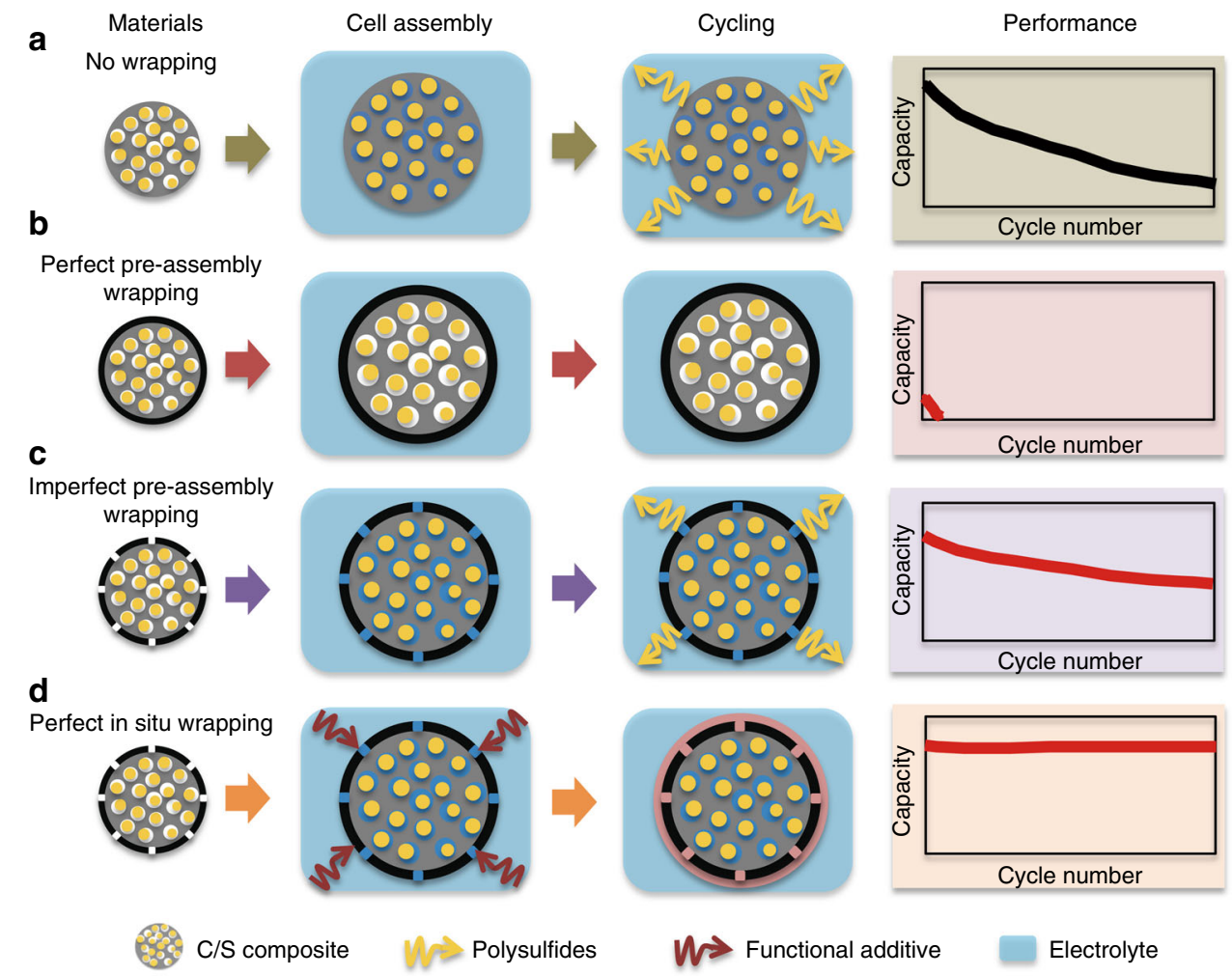

Fig. 1 Schematic illustration of the unique in situ wrapping strategy for lithium-sulfur cathode structures. a The no wrapping case, which exhibits severe capacity decay during cycling. b Perfect wrapping of the $\mathrm{C} / \mathrm{S}$ materials prior to battery assembly, which exhibits poor overall performance due to the lack of electrolyte in the active material. c Imperfect wrapping of the cathode material prior to battery assembly, which exhibits improved cycle stability compared with the no wrapping case. $\mathbf{d}$ Perfect post-assembly in situ wrapping of the cathode material, which exhibits ideal cycle stability using a blocking polysulfide shuttle while allowing for electrolyte infiltration in the active material 
specific capacity of $1246 \mathrm{mAh} \mathrm{g}^{-1}$ at $0.25 \mathrm{C}\left(1 \mathrm{C}=1672 \mathrm{~mA} \mathrm{~g}^{-1}\right)$ and a Coulombic efficiency of $98.2 \%$ over 100 cycles. Importantly, the capacity decay of the cell at the end of 1000 cycles is as small as $0.030 \%$ and $0.034 \%$ per cycle at $1 \mathrm{C}$ and $2 \mathrm{C}$, respectively.

\section{Results}

In situ wrapping and materials characterization. For a demonstration of the in situ wrapping strategy, nanoporous CMK-3 was selected as a model carbon host for the $\mathrm{C} / \mathrm{S}$ composite active materials. The CMK-3/S composite cathode material has been shown to be highly promising for $\mathrm{Li}-\mathrm{S}$ batteries but suffers from an unfavorable polysulfide shuttle effect ${ }^{14}$. In our experiment, the CMK-3/S composite was first prepared according to a procedure from the literature via the melt-diffusion method ${ }^{14}$. A polyacrylonitrile (PAN) layer was grown on CMK-3/S via free radical polymerization of acrylonitrile. The PAN layer was then sulfurized by mixing CMK-3/S@PAN with sublimed sulfur and heating to $300^{\circ} \mathrm{C}$ in a sealed tube. The resulting CMK-3/S@sulfurized PAN (CMK-3/S@PANS) was assembled into coin cells as the cathode material. Triphenylphosphine (TPP) was added into the DOL/DME electrolyte (1:1, v/v with $1 \mathrm{M}$ LiTFSI). TPP was reported to spontaneously react with the sulfur species, including $\mathrm{S}_{8}$ and sulfides, to yield triphenylphosphine sulfide (TPS) (Supplementary Fig. 1a) ${ }^{18,19}$. The TPP additive in the electrolyte was thus expected to form a TPS coating layer on top of the PANS layer(CMK-3/S@PANS@TPS) while allowing for electrolyte infiltration into the interior of the composite particles (Fig. 2a). The reaction of TPP and sulfur in the ether-based solvent was characterized using nuclear magnetic resonance spectroscopy and was confirmed to produce TPS (detailed analysis of the reaction between TPP and different components of the cell can be found in Supplementary Fig. 2).

The multilayered core-shell structure of the composite particle was characterized utilizing transmission electron microscopy (TEM). The original CMK-3/S particles displayed a clean surface (Fig. 2b). A coating layer with a thickness of $\sim 40 \mathrm{~nm}$ or less was clearly identified in the TEM images of the CMK-3/S@PANS particles (Fig. 2c). After soaking in TPP-added electrolyte, an additional coating layer $\sim 15 \mathrm{~nm}$ was observed in the CMK-3/S@PANS@TPS sample (Fig. 2d). Note that the CMK-3/S@PANS@TPS sample for TEM characterization was prepared by pressing the CMK-3/S@PANS powder into a tablet without binder. Then, the tablet was soaked in the electrolyte in an assembled coin cell, which produced a new layer from the in situ reaction inside the cell. Additional TEM images of the fresh cells can be found in Supplementary Fig. 3 .

The chemical composition of the wrapping layers was analyzed using X-ray photoelectron spectroscopy (XPS). The CMK-3/S sample showed two peaks at $\sim 165.3$ and $164.1 \mathrm{eV}$ in the $S 2 p$ region, which is indicative of $S_{8}$ molecules infused within the nanochannels of the CMK-3 host (Fig. 2e) ${ }^{20}$. In contrast, additional double peaks at $\sim 163.5 \mathrm{eV}$ and $162.3 \mathrm{eV}$ were observed in the S 2p spectrum of the CMK-3/S@PANS sample (Fig. 2f). This peak is characteristic of short-chain sulfides that are covalently bonded to the cyclized PAN backbones (Supplementary Fig. 1b) ${ }^{21}$. Note that the CMK-3/S@PANS@TPS sample also displayed similar double peaks at $\sim 163.3 \mathrm{eV}$ and $162.1 \mathrm{eV}$ (Fig. 2g). Although these peaks can be assigned to the $\mathrm{P}=\mathrm{S}$ functional group of TPS according to a previous report (Supplementary Fig. 1a) ${ }^{22}$, it is difficult to distinguish the two different species from the $S 2 \mathrm{p}$ spectrum. However, a $\mathrm{P} 2 \mathrm{p}$ peak that corresponds to TPS $(\sim 132.7 \mathrm{eV})$ rather than TPP $(\sim 131.0$ eV) was observed in the CMK-3/S@PANS@TPS sample (Fig. 2j), whereas there was no P signal in either the CMK-3/S@PANS or
CMK-3/S samples (Fig. 2h, i). These results confirm that the TPP additive in the electrolyte reacted with the PANS and a TPS layer was formed (a complete list of XPS peaks is included in Supplementary Table 1).

The multilayered core-shell structure was further confirmed by energy-dispersive X-ray spectroscopy (EDX), for which the instrument was equipped in the TEM system. The EDX line profile along the dashed arrow in Fig. 2k exhibits an increasing $\mathrm{S}$ content from the outside to the inside, a high $\mathrm{P}$ content in the outermost layer (from elemental P in the TPS layer) with a decreasing trend towards inside, and a $\mathrm{N}$ content that started from the middle layer (from elemental $\mathrm{N}$ in the PANS layer). These data indicate the successful construction of the multishelled structure via in situ wrapping.

The X-ray diffraction (XRD) patterns of all three samples showed broad bands rather than the standard peaks of $S_{8}$, which suggest that the sizes of $S_{8}$ particles are $\sim 5 \mathrm{~nm}$ or less (Supplementary Fig. $4 \mathrm{a})^{23}$. Considering that the channel width in CMK-3 was $2-5 \mathrm{~nm}$, this hypothesis is highly plausible. The synthesis of CMK-3/S@PANS caused a slight aggregation of CMK-3/S particles to form secondary particles approximately several micrometers in size, as shown in the scanning electron microscopy (SEM) images (Supplementary Fig. 4b). EDX mapping of the same area indicates an even distribution of $\mathrm{C}$, $\mathrm{S}, \mathrm{N}$, and $\mathrm{P}$ elements at this length scale (Supplementary Fig. 4c). Thermal gravimetric analyses (TGA) showed that the sulfur content in the CMK-3/S@PANS@TPS composite was 68 wt\% (Supplementary Fig. 5).

Improved battery performance. Figure 3 presents the electrochemical performance of the batteries with the in situ wrapped cathode materials. Control cells were assembled with CMK-3/S and CMK-3/S@PANS. Evidently, the batteries demonstrated different discharging behaviors (Fig. 3a). Two main discharge plateaus at $\sim 2.3$ and $2.1 \mathrm{~V}$ (vs $\mathrm{Li}^{+} / \mathrm{Li}$ ) that were observed in the CMK-3/S cells, which is typical of $\mathrm{Li}-\mathrm{S}$ cells, were assigned to the reduction of $S_{8}$ to higher-order polysulfides and the reduction of higher-order polysulfides to lower-order polysulfides and eventually $\mathrm{Li}_{2} \mathrm{~S}$, respectively ${ }^{3}$. A third plateau at $1.7 \mathrm{~V}$ appeared in the CMK-3/S@PANS cell, which is related to the reduction of short sulfide chains that were linked to the PAN backbones $^{24}$. In the CMK-3/S@PANS@TPS cell, the capacity of the $1.7 \mathrm{~V}$ discharge plateau was significantly reduced compared with the CMK-3/S@PANS cell. This result confirms that the short-chain sulfide on the PAN backbone was partially consumed in the reaction with TPP. The PANS short-chain sulfide reduction peak in the cyclic voltammetry $(\mathrm{CV})$ data almost disappeared in the CMK-3/S@PANS@TPS cell (Supplementary Fig. 6), which also corroborated the reaction of PANS with TPP. Note that the discharge plateau at $\sim 2.1 \mathrm{~V}$ slightly decreased with TPS wrapping in the initial cycle. However, the flat plateau returned in the third cycle and remained unchanged in the following cycles (Supplementary Fig. 7a), which may be related to sulfur activation. $\mathrm{Li}_{2} \mathrm{~S}$ preferred to deposit on locations with better electronic conductivity and electrolyte contact during the cycling. Hence, the polarization decreased with cycling ${ }^{16,25}$.

Importantly, the CMK-3/S@PANS@TPS cell exhibited remarkably enhanced cycling stability. The results demonstrated a Coulombic efficiency of $98.2 \%$ and capacity retention ratio of 89\% after 100 cycles. For comparison, the CMK-3/S@PANS battery displayed a Coulombic efficiency of $90.3 \%$ and a capacity retention ratio of $67 \%$, and the CMK-3/S battery displayed a Coulombic efficiency of $80.2 \%$ and a capacity retention ratio of $60 \%$ after 100 cycles (Fig. 3b). Even after 500 cycles, the CMK-3/S@PANS@TPS battery showed a capacity retention ratio 
of $\sim 83.5 \%$ (decay rate of $\sim 0.033 \%$ per cycle) (Fig. 3e). Additional discharge-charge curves at later cycles can be found in Supplementary Fig. 7b. The CMK-3/S@PANS@TPS battery also showed excellent rate performance with discharge capacities of 1246, 1078, 995, and $915 \mathrm{mAh} \mathrm{g}^{-1}$ at $0.25 \mathrm{C}, 0.5 \mathrm{C}, 1 \mathrm{C}$ and $2 \mathrm{C}$ rates, respectively. When the current returned to $0.25 \mathrm{C}$, the reversible capacity was $1042 \mathrm{mAh} \mathrm{g}^{-1}$ (Fig. 3c).
The CMK-3/S@PANS@TPS battery achieved the best performance at $2 \mathrm{wt} \%$ TPP concentration in the electrolyte. Higher TPP content resulted in enhanced cycling stability but caused a slight decrease in capacity since $S_{8}$ inside the CMK-3 matrix may also react with excessive TPP (Fig. 3d). Figure 3e displays the extended cycle performance of the CMK-3/S@PANS@TPS cell. When cycled at $1 \mathrm{C}$, the battery exhibited an initial specific capacity of $994 \mathrm{mAh} \mathrm{g}^{-1}$ and a specific capacity after 1000 cycles was $\sim 698$ a

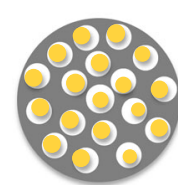

b
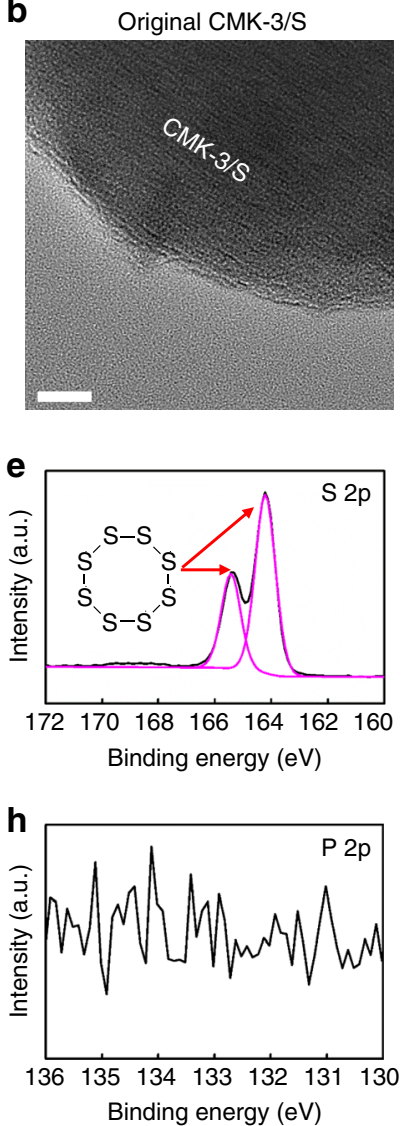

C
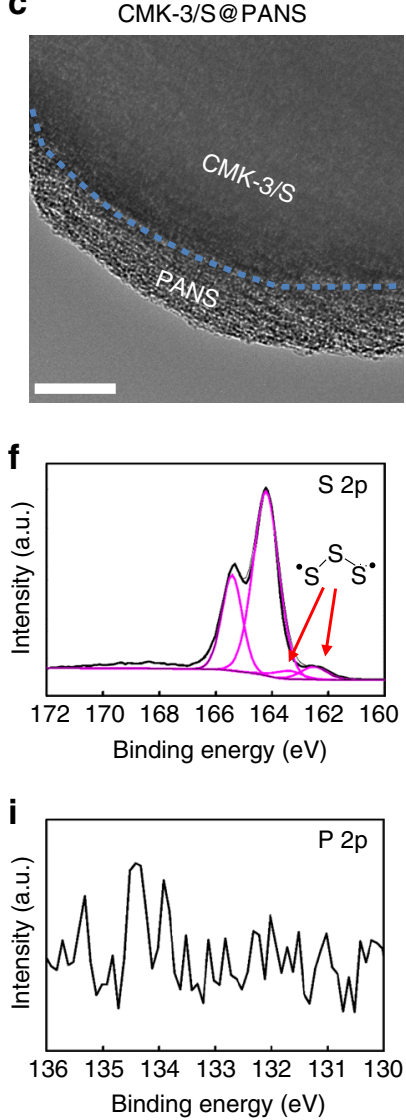

TPP in electrolyte In situ wrapping

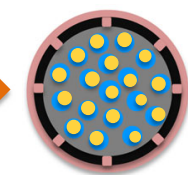

d

CMK-3/S@PANS@TPS
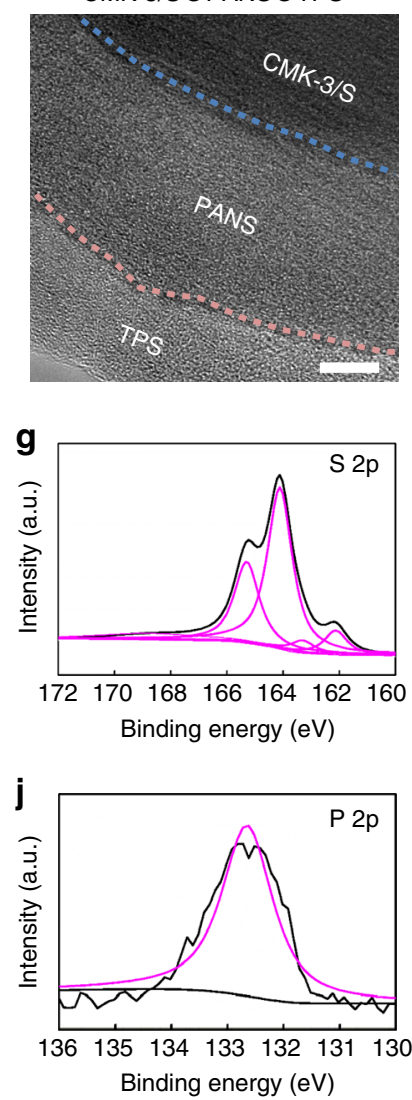

$\mathbf{k}$

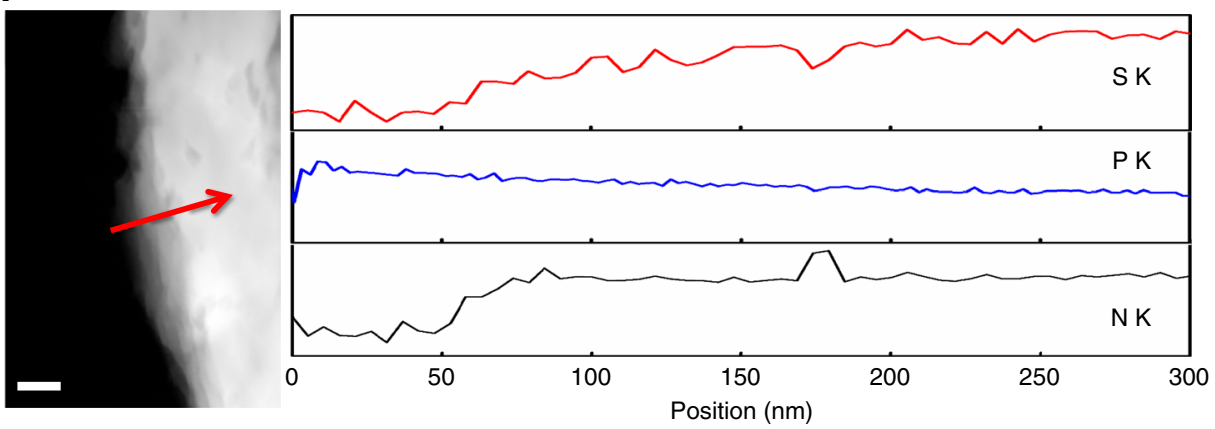

Fig. 2 Materials characterization. a Schematic illustration of the in situ wrapping process. TEM images of b CMK-3/S, scale bar: 20 nm, c CMK-3/S@PANS, scale bar: 50 nm and d CMK-3/S@PANS@TPS particles, scale bar: 10 nm. $\mathrm{S}_{2 \mathrm{p}}$ XPS spectra of e CMK-3/S, (f) CMK-3/S@PANS, and $\mathbf{g}$

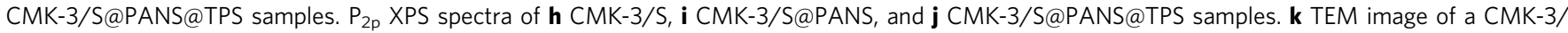
S@PANS@TPS particle and the EDX elemental mapping across the multi-layered core-shell composite particle along the dashed line with the arrow pointing in the direction from 0 to $300 \mathrm{~nm}$ in the position, scale bar: $100 \mathrm{~nm}$ 
a

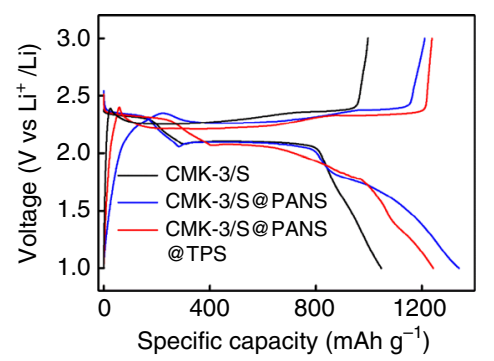

C

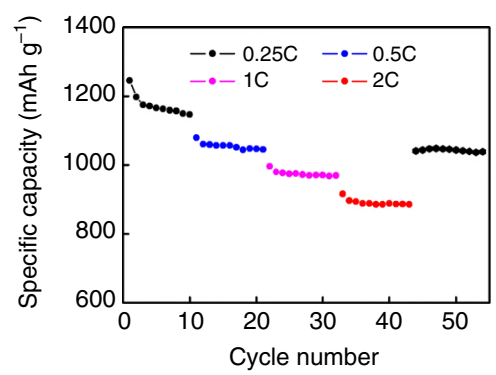

b

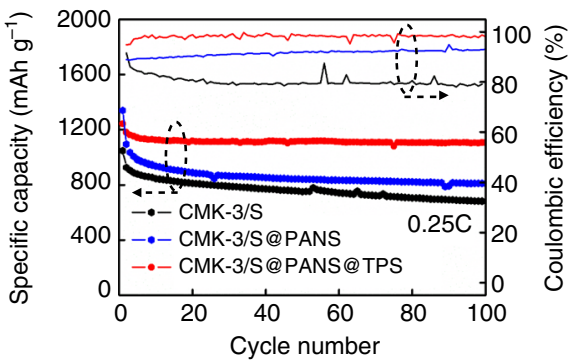

d

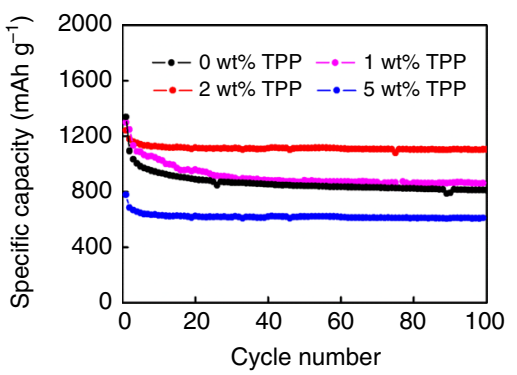

e

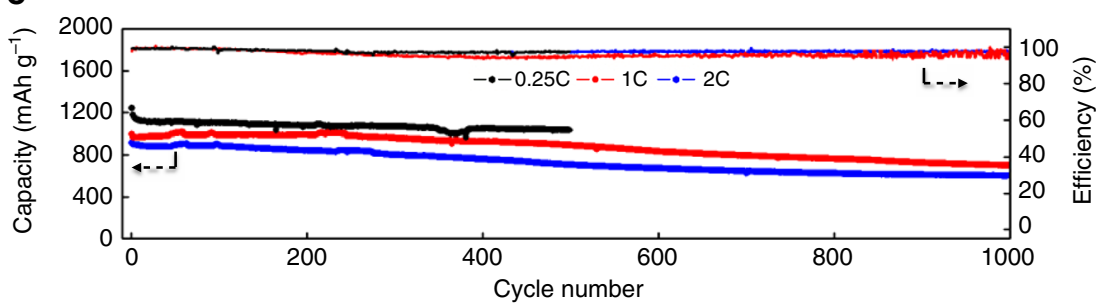

Fig. 3 Electrochemical performance of the lithium-sulfur batteries. a Initial galvanostatic cyclic curve of CMK-3/S, CMK-3/S@PANS, and CMK-3/ S@PANS@TPS cathodes. b Cycling performance of CMK-3/S, CMK-3/S@PANS,CMK-3/S@PANS@TPS cathodes at 0.25 C (1 C=1672 mA g $\left.{ }^{-1}\right)$.c Rate performance of the CMK-3/S@PANS@TPS cathode.d Cycling performance of CMK-3/S@PANS@TPS cathode with various TPP contents in the electrolyte at 0.25 C. e Extended cycling performance of CMK-3/S@PANS@TPS cathodes at 0.25 C, 1C, and 2 C

$\mathrm{mAh}^{-1}$ with a stabilized $96.2 \%$ Coulombic efficiency. When cycled at $2 \mathrm{C}$, the initial capacity decreased to $917 \mathrm{mAh} \mathrm{g}^{-1}$ and the specific capacity after 1000 cycles was $\sim 598 \mathrm{mAh} \mathrm{g}^{-1}$ with a 98.4\% Coulombic efficiency. The overall capacity decay rates were $0.030 \%$ and $0.034 \%$ at $1 \mathrm{C}$ and $2 \mathrm{C}$, respectively. TEM images of the material from the cycled cells showed that the two-layered wrapping structure was stable after long-term cycling (Supplementary Fig. 8).

Suppressed polysulfide shuttling. The sophisticated control of pore sizes in the in situ wrapped multilayered core-shell structure plays a critical role in battery performance enhancement. Figure 4a displays the pore size distribution of the samples obtained with the non-local density functional theory analysis of Brunauer-Emmett-Teller (BET) measurements. $\mathrm{CMK}-3 / \mathrm{S}$ was rich in mesopores $\sim 1.2$ and $2.5 \mathrm{~nm}$ in size, and CMK-3/S@PANS has similar mesopores along with a new micropore distribution at $\sim 0.8 \mathrm{~nm}$. In addition, the pore size distribution analysis on pure PANS also shows the coexistence of both micro- and mesopores. The presence of micro- and mesopores in the structure allows the infiltration of electrolyte in the cell and the storage of electrolyte in the pores. In contrast, these pore structures virtually disappear following the in situ wrapping reaction. The BET measurements show that a small distribution of micropores that range from 0.5 to $0.6 \mathrm{~nm}$ was present in the CMK-3/S@PANS@TPS material for $\mathrm{N}_{2}$ adsorption. The extremely low pore volume suggests that these pores are not through-holes.

The change in pore structure has significant consequences in ion and mass transport within the cathode. First, Li ions can pass through the TPS layer due to its small ionic radius of $0.69 \AA^{26}$. In addition, previous reports indicate that TPP and its derivatives (such as triphenylphosphite) facilitated the formation of the cathode electrolyte interface due to the electron-rich phenyl structure (Supplementary Fig. 9), which enables efficient $\mathrm{Li}^{+}$ transportation 27,28 . Similar structural moieties, which are also richly present in the TPS layer, facilitated $\mathrm{Li}^{+}$conduction (Supplementary Fig. 9).

Second, polysulfide ions and solvent molecules in the electrolyte are blocked by the TPS layer. A recent study estimates that the size of lithium polysulfides $\left(\operatorname{Li}_{2} \mathrm{~S}_{n}, 2<n \leq 8\right)$ were $>0.8 \mathrm{~nm}^{29}$. Another recent study demonstrated that polysulfides are incapable of permeating pores with sizes $<0.9 \mathrm{~nm}^{30}$. The sizes of DME and DOL solvent molecules were also calculated, as shown in Supplementary Fig. 10. The sizes of single DME and DOL molecules are 7.57 and $3.55 \AA$, respectively. As the pores in TPS are not through-holes, the solvent molecules cannot pass through the TPS layer, even though the DOL size appeared to be $<0.5-0.6 \mathrm{~nm}$ pore size.

Thus, the in situ-formed TPS wrapping layer can successfully block polysulfide shuttling. Furthermore, the cathode reaction 
a

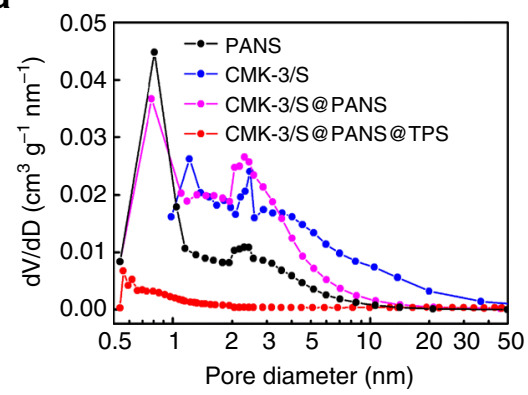

C

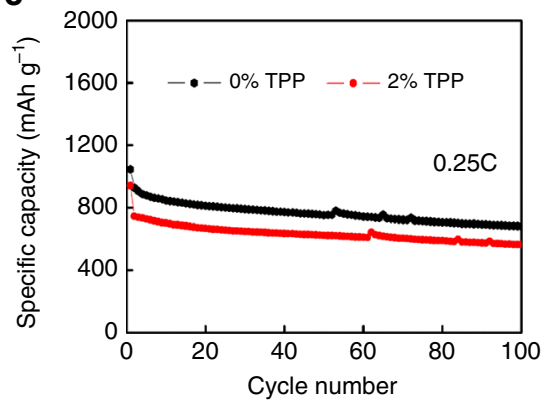

b

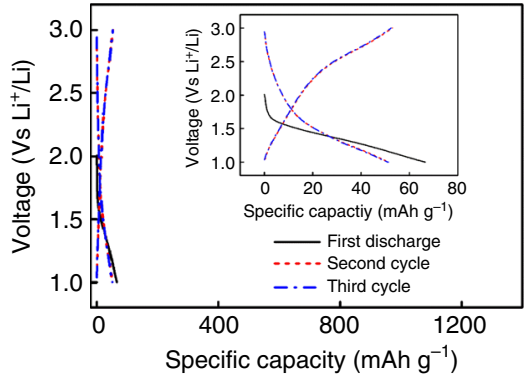

d

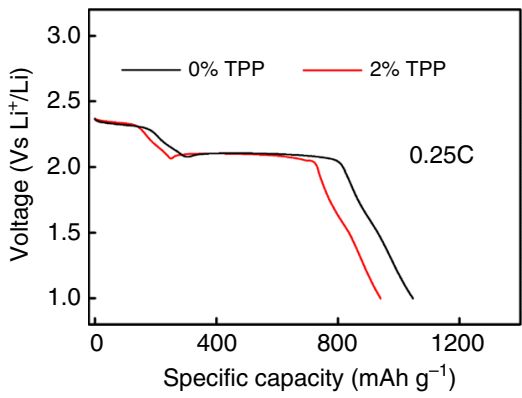

Fig. 4 Pore size distribution and electrochemical performance of the controlled samples. a The pore size distribution of PANS, CMK-3/S, CMK-3/S@PANS, and CMK-3/S@PANS@TPS materials.b Galvanostatic discharge/charge curves of the control cell with the TPS layer wrapped on CMK-3/S@PANS before cell assembly. The inset figure shows the enlarged discharge/charge curves. c Cycling performance of the CMK-3/S cells with and without TPP in the electrolyte. d The initial discharge curves of the CMK-3/S cells with and without TPP in the electrolyte

may still occur. In cycling, $\mathrm{Li}^{+}$ions may desolvate at the outer surface of the TPS layer and then pass through the TPS layer. As the ions move into the interior of the TPS wrapping layer, they may either quickly react with sulfur species or become resolvated in the electrolyte inside the active material. This notion is strongly supported by the control experiment using a perfect preassembled TPS coating as described below. A different CMK-3/ S@PANS@TPS material was prepared by soaking the CMK-3/ S@PANS material in a TPP-containing electrolyte without $\mathrm{Li}$ salt (2 wt\% TPP in DOL/DME) and then vacuum drying the product to remove the residual liquid. The material was then assembled into coin cells. Remarkably, the specific capacity was as small as $\sim 70 \mathrm{mAh} \mathrm{g}^{-1}$ in the initial cycle, and there was no distinguishable discharge plateau characteristic of the polysulfides. Similar behavior persisted in the following cycles (Fig. 4b). These results indicate that the TPS layer effectively isolated the active material from the electrolyte. Thus, this control experiment demonstrated the case of a "perfect preassembled wrapping," as illustrated in Fig. 1b.

Note that the PANS wrapping layer was necessary in this design to provide a substrate for the growth of the TPS layer. In another control experiment, in which the CMK-3/S material was assembled into a coin cell with the TPP-containing electrolyte, there was no improvement in cycle stability, and the specific capacity was slightly reduced, which was presumably due to the loss of active sulfur material to the reaction with added TPP (Fig. 4c, d). If TPP polymerized on the membrane or Li metal to form a wrapping layer, which would have positive effects on the cell performance, then the performance of the cell with CMK-3/S and 2\% TPP in the electrolyte will also improve. Therefore, the improvement in cell performance originated from the in situ reaction between TPP and the PANS layers.

Electrochemical impedance spectroscopy measurements show depressed semicircles in the high- and medium-frequency regions (representing charge transfer resistance ${ }^{31,32}$ ), which were followed by an inclined line in the low-frequency region (Supplementary Fig. 11). The smallest semicircle for the CMK-3/S@PANS@TPS cell suggests that the in situ-formed TPS layer may reduce the charge transfer resistance and contribute to the enhanced performance. This impedance reduction resulted from electron-rich phenyl structural moieties in TPS, which enhanced $\mathrm{Li}^{+}$transport ${ }^{27,28}$. Note that there are additional semicircles in the CMK-3/S@PANS and CMK-3/ S@PANS@TPS samples compared with the CMK-3/S cathode. This behavior can be caused by the coating layers, which may provide added impedance for lithium ion diffusion into the CMK-3 core from the outside electrolyte. Similar behavior has been reported in which the encapsulation of active materials leads to an additional semicircle and extra impedance for lithium ion diffusion $^{33}$.

The effect of the in situ-formed TPS layer on polysulfide shuttling was proven by a direct shuttle current measurement and a glass cell visual test. Narayanan et al. reported a simple and direct measurement to quantify the polysulfide shuttling process in $\mathrm{Li}-\mathrm{S}$ batteries ${ }^{34}$. Generally, the potential of the $\mathrm{Li}-\mathrm{S}$ cathode steadily decreased because soluble polysulfides continuously arrived at the cathode from the anode (i.e., the polysulfide shuttling process). To maintain a constant cathode potential, a Faradic current was needed to balance/oxidize the polysulfide flux, which is measured as the shuttle current ${ }^{34}$. Following the reported protocol (also see details in the method section) ${ }^{34}$, the shuttle current measurements were made, and the results are displayed in Fig. 5a. Similar to the literature, the current-time profiles presented an initial transient that was associated with the small difference between the open-circuit voltage and hold voltage and then quickly approached a steady-state value that was directly attributed to the shuttle current ${ }^{34}$. The shuttle current of the CMK-3/S@PANS@TPS cell was significantly smaller than the other two cells without the TPS layer, which indicates significantly suppressed polysulfide shuttling. In the positive 
a

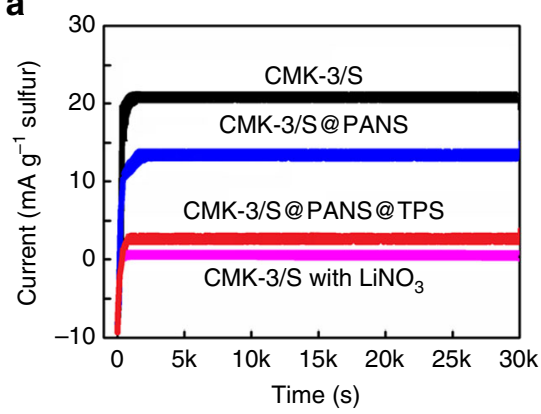

b

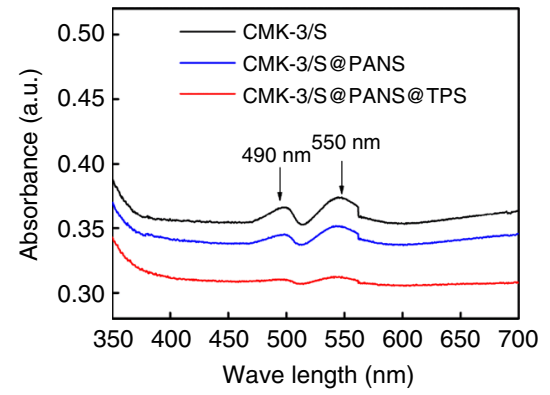

e
C

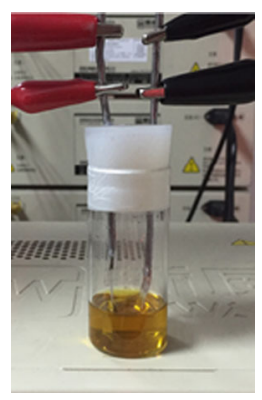

CMK-3/S d

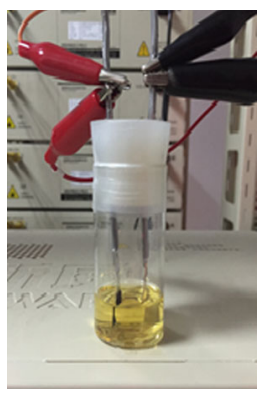

CMK-3/S@PANS

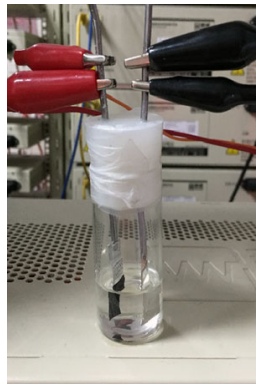

CMK-3/S@PANS@TPS

Fig. 5 Suppression of polysulfide shuttling using in situ wrapping. a Shuttle current profiles of CMK-3/S, CMK-3/S@PANS, and CMK-3/S@PANS@TPS cathodes and a CMK-3/S cathode in an electrolyte with $1 \mathrm{wt} \%$ lithium nitrate (measured at a constant holding potential of $2.28 \mathrm{~V}$ ). b UV-vis absorption spectra of the electrolytes after 20 cycles with various cathodes in glass cells at 0.1 C. c-e Photographs of CMK-3/S, CMK-3/S@PANS, and CMK-3/S@PANS@TPS cathodes after 20 cycles at 0.1C in sealed glass cells

control with the CMK-3/S cell that contained $1 \mathrm{wt} \%$ lithium nitrate in the electrolyte, the shuttle current was completely blocked. Lithium nitrate has been known to suppress the polysulfide shuttle by forming a passivation film on the $\mathrm{Li}$ anode surface $^{35}$. However, lithium nitrate is a consumptive additive, and thus, the decline in cell capacity becomes unavoidable in continuous cycling operations ${ }^{36}$.

The suppression of polysulfide shuttling with the in situformed TPS layer was also visualized in glass cells. In the photographs in Fig. $5 c-e$, cathodes with various materials were cycled in sealed glass cells against a $\mathrm{Li}$ anode to simulate the reactions in the coin cells. After 20 cycles at $0.1 \mathrm{C}$, the polysulfide intermediates gradually dissolved and diffused into the electrolyte, which resulted in a yellow color that depended on the concentration of dissolved polysulfides ${ }^{12,37}$. The CMK-3/S cell displayed a deep golden color, which indicated the most severe polysulfide dissolution. The CMK-3/S@PANS cell showed a light yellow color, which suggested that PANS could partially suppress polysulfide shuttling. In contrast, no obvious color change was observed in the CMK-3/S@PANS@TPS cell, which indicated highly effective blocking of the shuttling effect by the TPS layer. The UV-vis spectra of the electrolytes demonstrated absorption characteristics of $\mathrm{Li}_{2} \mathrm{~S}_{n}(2<n \leq 3)$ and $\mathrm{Li}_{2} \mathrm{~S}_{n}(4 \leq n \leq 6)$ at $\sim 490$ $\mathrm{nm}$ and $550 \mathrm{~nm}$, respectively (Fig. 5b) ${ }^{38}$. Compared with the other two samples, the electrolyte from the CMK-3/ S@PANS@TPS cell clearly showed the weakest absorption, which suggests the smallest polysulfide concentration. These results are consistent with the shuttle current measurements and indicated that the in situ-formed TPS wrapping layer was responsible for suppressing the shuttling effect.

\section{Discussion}

In summary, an in situ wrapping strategy for the Li-S cathode was developed. In conventional approaches, in which the cathode material with a wrapping layer was first synthesized and then assembled into batteries, there is a trade-off between electrolyte infiltration and blocking polysulfide diffusion. The in situ wrapping approach solves this trade-off by first allowing the electrolyte to infuse into the interior of the porous cathode material and then closing the pores by a surface reaction with a special additive in the electrolyte. The idea was realized via the reaction between PANS and TPP. The in situ wrapping mechanism has been verified using characterization tools and control experiments, and the polysulfide shuttle blocking effect has been directly proven by shuttle current measurements. Batteries with in situ wrapped cathode materials showed greatly improved cycling stability, i.e., a decay rate of $\sim 0.030 \%$ per cycle within the 1000 cycles at a current of $1 \mathrm{C}$, which is far slower than that of typical cathode materials coated using conventional pre-assembly wrapping approaches.

This in situ wrapping strategy provides conceptually new opportunities for $\mathrm{Li}-\mathrm{S}$ cathode materials. Considering versatile in situ wrapping reaction types, including photochemical and electrochemical methods, there are unlimited possibilities in the molecular design of in situ wrapping reagents. Furthermore, there is no doubt that the stability of $\mathrm{Li}-\mathrm{S}$ cathodes can be further optimized. Importantly, this approach is in principle applicable to all $\mathrm{C} / \mathrm{S}$ composites and is thus compatible with scale-up attempts in the engineering of large-capacity pouch cells.

\section{Methods}

Chemicals. Sulfur powder (99.9\%) was purchased from Sigma Aldrich. CMK-3 was acquired from XFNANO Materials Tech Co., Ltd. (Nanjing, China). 2,2'-Azobisisobutyronitrile (AIBN), dimethyl sulfoxide (DMSO) and triphenylphosphine (TPP) were purchased from Sigma Chemical Co. and used without purification. Acrylonitrile (AN) monomer was purchased from Aladdin Reagent Co., Ltd. (Shanghai, China). Prior to use, AN was washed with 3\% phosphoric acid and 5\% sodium hydroxide to remove the inhibitor, then repeatedly washed with deionized water, dried over calcium chloride and stored in a refrigerator. 
Synthesis of CMK-3/S composite. CMK-3 and S were mixed at a 3:7 mass ratio using ground milling. The mixture was sealed and heated under argon up to $155^{\circ} \mathrm{C}$ at a heating rate of $10^{\circ} \mathrm{C} \mathrm{min}^{-1}$. The mixture was maintained at $155^{\circ} \mathrm{C}$ for $12 \mathrm{~h}$ to obtain the CMK-3/S composite.

Synthesis of PANS. An acrylonitrile monomer $(10 \mathrm{ml})$ and AIBN $(75 \mathrm{mg})$ were added to a mixed solvent of deionized water $(25 \mathrm{ml})$ and DMSO $(25 \mathrm{ml})$. The solution was stirred under a $\mathrm{N}_{2}$ atmosphere for $3 \mathrm{~h}$ at $65^{\circ} \mathrm{C}$ to carry out the polymerization. After polymerization, the milky white product was collected by centrifugation and washed with ethanol. The product was vacuum dried at $60^{\circ} \mathrm{C}$ for $24 \mathrm{~h}$ to produce PAN. PAN $(100 \mathrm{mg})$ and S $(300 \mathrm{mg})$ were ground together, then sealed and heated under argon at $300^{\circ} \mathrm{C}$ (with a heating rate of $10^{\circ} \mathrm{C} \mathrm{min}^{-1}$ ) for $3 \mathrm{~h}$ to obtain PANS ${ }^{39}$.

Synthesis of CMK-3/S@PANS. The as-prepared CMK-3/S composite $(0.5 \mathrm{~g})$ was dispersed in a mixed solution of deionized water $(25 \mathrm{ml})$ and DMSO $(25 \mathrm{ml})$ under ultrasonication $(60 \mathrm{~W}$, Sonics \& Materials, Inc., USA) for $10 \mathrm{~min}$ Acrylonitrile monomer $(10 \mathrm{ml})$ and AIBN $(75 \mathrm{mg})$ were then added. The mixed solution was stirred under a $\mathrm{N}_{2}$ atmosphere for $3 \mathrm{~h}$ at $65^{\circ} \mathrm{C}$ to conduct the polymerization. The resulting solid was collected by centrifugation and washed with ethanol after polymerization. The product was vacuum dried at $60^{\circ} \mathrm{C}$ for $24 \mathrm{~h}$ to yield CMK-3/S@PAN. Next, CMK-3/S@PAN and S (mass ratio PAN:S, 1:3) were ground together, then sealed and heated under argon at a rate of $10^{\circ} \mathrm{C} \mathrm{min}{ }^{-1}$ up to $300^{\circ} \mathrm{C}$. The mixture was maintained at $300^{\circ} \mathrm{C}$ for $3 \mathrm{~h}$ to obtain CMK-3/S@PANS ${ }^{39}$.

Preparation of various cathodes. The CMK-3/S composite was mixed with carbon black and the water-based binder LA-132 (Chengdu Indigo Power Sources Co., Ltd., China) with a weight ratio of 7:2:1 in deionized water with vigorous stirring for $24 \mathrm{~h}$ to form a cathode slurry. The slurry was coated onto an aluminum collector and baked at $60^{\circ} \mathrm{C}$ for $24 \mathrm{~h}$ to form the CMK-3/S cathode. The CMK-3/S@PANS cathode used the same procedure, except that CMK-3/S was replaced by CMK-3/S@PANS. The mass loading of sulfur in the cathode was $\sim 2.1 \mathrm{mg} \mathrm{cm}^{-2}$.

Cell assembly. Coin-type (CR2025) cells were fabricated by sandwiching a porous polypropylene separator between a cathode that contained the active materials and a lithium metal foil in a high-purity argon-filled glove box. Unless stated otherwise, the electrolyte used in the CMK-3/S and CMK-3/S@PANS cells was 1 M LiTFSI in a solvent mixture of DOL/DME (1:1, v/v). The electrolyte used in the CMK-3/ S@PANS@TPS cell was 1 M LiTFSI in a solvent mixture of DOL/DME (1:1 v/v) with TPP additives at $1 \mathrm{wt} \%, 2 \mathrm{wt} \%$, or $5 \mathrm{wt} \%$ concentrations. When assembling the cells for the in situ wrapping reaction, a drop of $\sim 10 \mu \mathrm{l}$ blank electrolyte without TPP was added to the CMK-3/S@PANS cathode to initially wet the cathode material, and the electrolyte with $2 \mathrm{wt} \%$ TPP was then added to complete the cell assembly. Then, the cell was then maintained at room temperature for $6 \mathrm{~h}$ before electrochemical testing.

Characterizations and electrochemical testing. TEM images were recorded using a Tecnai G2 F20 S-TWIN system that was equipped with energy dispersive $\mathrm{X}$-ray (EDX) microanalysis capabilities at $200 \mathrm{kV}$. SEM images were recorded using an FEI Quanta 400 FEG microscope that was also equipped with EDX capabilities. $\mathrm{X}$-ray photoelectron spectroscopy was performed on an SSI S-Probe XPS spectrometer. XRD patterns were recorded using a Bruker D8 diffractometer with $\mathrm{Cu} \mathrm{K} \alpha$ radiation. TGA was performed using a Seiko TG/DTA 6300 instrument under $\mathrm{N}_{2}$ protection at a heating rate of $10^{\circ} \mathrm{C} \mathrm{min}^{-1}$. The pore size distribution of the materials was measured employing the Brunauer-Emmett-Teller (BET) method using nitrogen adsorption and desorption isotherms on a 3H-2000PS2 system (Beishide Instrument Technology Co., Ltd., China). The pore size distribution was calculated using the non-local density functional theory model. UV-visible absorption spectroscopy was performed on a Lambda-750 spectrometer (Pekin-Elmer) at room temperature at wavelengths that ranged from 800 to 300 $\mathrm{nm}$. Each sample that contained $20 \mu \mathrm{l}$ of electrolyte from the glass cell was diluted to $2 \mathrm{ml}$ with DOL/DME (1:1, v/v). Galvanostatic discharge-charge tests were performed on a battery test system (Neware, Neware Technology Co., Ltd., Shenzhen, China) from 1 to $3 \mathrm{~V}$. Cyclic voltammetry (CV) scans were performed with a coin cell on a CHI 660 C electrochemical workstation (CHI Instruments, Inc.) at a scan rate of $0.1 \mathrm{mV} \mathrm{s}^{-1}$. Electrochemical impedance spectroscopy was conducted with a coin cell-based two-electrode configuration, in which lithium served as both the counter electrode and reference electrode. The frequency range for the impedance study was $100 \mathrm{kHz}$ to $0.1 \mathrm{~Hz}$. The visible electrochemical cell test was carried out in two-electrode glass cells. A $1 \mathrm{~cm}^{2}$ cathode and $10 \mathrm{ml}$ of electrolyte were used in each cell. The glass cells were assembled in a glove box to eliminate the influence of water and oxygen. For the shuttle current test, the cells were initially discharged and charged three times at a $0.05 \mathrm{C}$ rate. Then, the cells were charged to $2.7 \mathrm{~V}$ and allowed to rest in an open-circuit condition for $10 \mathrm{~min}$. Next, the cell voltage was maintained at the open-circuit voltage $(2.28 \mathrm{~V})$, and the current that was applied to the cell was observed for at least $1 \mathrm{~h}$ until a steady-state value was attained. This steady-state current was recorded as the shuttle current ${ }^{34}$.
Data availability. The supporting data of this study are available from the corresponding authors upon request.

Received: 4 December 2016 Accepted: 18 July 2017

Published online: 07 September 2017

\section{References}

1. Yin, Y. X., Xin, S., Guo, Y. G. \& Wan, L. J. Lithium-sulfur batteries: electrochemistry, materials, and prospects. Angew. Chem. Int. Ed. 52, 13186-13200 (2013).

2. Bruce, P. G., Freunberger, S. A., Hardwick, L. J. \& Tarascon, J. M. Li- $\mathrm{O}_{2}$ and Li-S batteries with high energy storage. Nat. Mater. 11, 19-29 (2012).

3. Zhang, S. S. Liquid electrolyte lithium/sulfur battery: fundamental chemistry, problems, and solutions. J. Power Sources 231, 153-162 (2013).

4. Mikhaylik, Y. V. \& Akridge, J. R. Polysulfide shuttle study in the Li/S battery system. J. Electrochem. Soc. 151, A1969-A1974 (2004).

5. Manthiram, A., Fu, Y., Chung, S. H., Zu, C. \& Su, Y. S. Rechargeable lithium-sulfur batteries. Chem. Rev. 114, 11751-11787 (2014).

6. Yang, Y. et al. Improving the performance of lithium-sulfur batteries by conductive polymer coating. Acs Nano 5, 9187-9193 (2011).

7. Xiao, L. et al. A soft approach to encapsulate sulfur: polyaniline nanotubes for lithium-sulfur batteries with long cycle life. Adv. Mater. 24, 1176-1181 (2012).

8. Jayaprakash, N., Shen, J., Moganty, S. S., Corona, A. \& Archer, L. A. Porous hollow carbon@sulfur composites for high-power lithium-sulfur batteries. Angew. Chem. Int. Ed. 50, 5904-5908 (2011).

9. Zhang, C., Wu, H. B., Yuan, C., Guo, Z. \& Lou, X. W. Confining sulfur in double-shelled hollow carbon spheres for lithium-sulfur batteries. Angew. Chem. Int. Ed. 124, 9730-9733 (2012).

10. Zhou, W., Yu, Y., Chen, H., DiSalvo, F. J. \& Abruna, H. D. Yolk-shell structure of polyaniline-coated sulfur for lithium-sulfur batteries. J. Am. Chem. Soc. 135 16736-16743 (2013).

11. Chen, H. W. et al. In-situ activated polycation as a multifunctional additive for Li-S batteries. Nano Energy 26, 43-49 (2016).

12. Chen, H. W. et al. Rational design of cathode structure for high rate performance lithium-sulfur batteries. Nano Lett. 15, 5443-5448 (2015).

13. Seh, Z. W. et al. Sulfur- $\mathrm{TiO}_{2}$ yolk-shell nanoarchitecture with internal void space for long-cycle lithium-sulfur batteries. Nat. Commun. 4, 1331-1337 (2013).

14. Ji, X., Lee, K. T. \& Nazar, L. F. A highly ordered nanostructured carbon-sulfur cathode for lithium-sulfur batteries. Nat. Mater. 8, 500-506 (2009).

15. Li, W. et al. Understanding the role of different conductive polymers in improving the nanostructured sulfur cathode performance. Nano Lett. 13 5534-5540 (2013).

16. $\mathrm{Hu}, \mathrm{H}$. et al. In situ polymerized PAN-assisted S/C nanosphere with enhanced high-power performance as cathode for lithium/sulfur batteries. Nano Lett. 15 5116-5123 (2015).

17. Chen, H. W. et al. Ultrafine sulfur nanoparticles in conducting polymer shell as cathode materials for high performance lithium/sulfur batteries. Sci. Rep. 3, 1910-1916 (2013).

18. Chung, W. J. et al. Elemental sulfur as a reactive medium for gold nanoparticles and nanocomposite materials. Angew. Chem. Int. Ed. 50, 11409-11412 (2011).

19. Bartlett, P. D. \& Meguerian, G. Reactions of elemental sulfur. I. The uncatalyzed reaction of sulfur with triarylphosphines1. J. Am. Chem. Soc. 78, 3710-3715 (1956).

20. Li, Z. et al. Insight into the electrode mechanism in lithium-sulfur batteries with ordered microporous carbon confined sulfur as the cathode. Adv. Energy Mater. 4, 1301473-1301480 (2014).

21. Fanous, J., Wegner, M., Grimminger, J., Andresen, Ä. \& Buchmeiser, M. R. Structure-related electrochemistry of sulfur-poly(acrylonitrile) composite cathode materials for rechargeable lithium batteries. Chem. Mater. 23, 5024-5028 (2011).

22. Morgan, W. E., Stec, W. J., Albridge, R. G. \& Van Wazer, J. R. pi.-Bond feedback interpreted from the binding energy of the " $2 \mathrm{p}$ " electrons of phosphorus. Inorg. Chem. 10, 926-930 (1971).

23. Chen, H. W. et al. Monodispersed sulfur nanoparticles for lithium-sulfur batteries with theoretical performance. Nano Lett. 15, 798-802 (2015).

24. Zhang, S. S. Sulfurized carbon: a class of cathode materials for high performance lithium/sulfur batteries. Front. Energy Res. 1, A1-A7 (2013).

25. Li, W. et al. High-performance hollow sulfur nanostructured battery cathode through a scalable, room temperature, one-step, bottom-up approach. Proc. Natl Acad. Sci. USA 110, 7148-7153 (2013). 
26. Palomares, V. et al. Na-ion batteries, recent advances and present challenges to become low cost energy storage systems. Energy Environ. Sci. 5, 5884-5901 (2012).

27. Xu, J., Hu, Y., Liu, T. \& Wu, X. Improvement of cycle stability for high-voltage lithium-ion batteries by in-situ growth of SEI film on cathode. Nano Energy 5, 67-73 (2014).

28. Jia, H. et al. TPPi as a flame retardant for rechargeable lithium batteries with sulfur composite cathodes. Chem. Commun. 50, 7011-7013 (2014).

29. Vijayakumar, M. et al. Molecular structure and stability of dissolved lithium polysulfide species. Phys. Chem. Chem. Phys. 16, 10923-10932 (2014).

30. Bai, S., Liu, X., Zhu, K., Wu, S. \& Zhou, H. Metal-organic framework-based separator for lithium-sulfur batteries. Nat. Energy 1, 16094-16099 (2016).

31. Deng, Z. et al. Electrochemical impedance spectroscopy study of a lithium/ sulfur battery: modeling and analysis of capacity fading. J. Electrochem. Soc. 160, A553-A558 (2013).

32. Wang, J. et al. Towards a safe lithium-sulfur battery with a flame-inhibiting electrolyte and a sulfur-based composite cathode. Angew. Chem. Int. Ed. 53, 10099-10104 (2014).

33. Shen, Y. B., Eltzholtz, J. R. \& Iversen, B. B. Controlling size, crystallinity, and electrochemical performance of $\mathrm{Li}_{4} \mathrm{Ti}_{5} \mathrm{O}_{12}$ nanocrystals. Chem. Mater. 25, 5023-5030 (2013).

34. Moy, D., Manivannan, A. \& Narayanan, S. R. Direct measurement of polysulfide shuttle current: a window into understanding the performance of lithium-sulfur cells. J. Electrochem. Soc. 162, A1-A7 (2014).

35. $\mathrm{Li}, \mathrm{W}$. et al. The synergetic effect of lithium polysulfide and lithium nitrate to prevent lithium dendrite growth. Nat. Commun. 6, 7436-7442 (2015).

36. Zhang, S. S. Role of $\mathrm{LiNO}_{3}$ in rechargeable lithium/sulfur battery. Electrochim. Acta 70, 344-348 (2012).

37. Fan, Q., Liu, W., Weng, Z., Sun, Y. \& Wang, H. Ternary hybrid material for highperformance lithium-sulfur battery. J. Am. Chem. Soc. 137, 12946-12953 (2015).

38. Patel, M. U. M. \& Dominko, R. Application of in operando UV/vis spectroscopy in lithium-sulfur batteries. Chemsuschem 7, 2167-2175 (2014).

39. Yin, L., Wang, J., Yang, J. \& Nuli, Y. A novel pyrolyzed polyacrylonitrilesulfur@MWCNT composite cathode material for high-rate rechargeable lithium/sulfur batteries. J. Mater. Chem. 21, 6807-6810 (2011).

\section{Acknowledgements}

We acknowledge funding support from Ministry of Science and Technology (Grant No. 2016YFA0200700), the "Strategic Priority Research Program" of the CAS (Grant No.
XDA09010600), the Natural Science Foundation of China (Grant Nos. 21625304, 21473242, 21273273, 61604167) and the Collaborative Innovation Center of Suzhou Nano Science and Technology. L.C. acknowledges the Natural Science Foundation of Jiangsu for support (BK20130006). We also thank Guotai Huarong Chemical New Material Co., Ltd. for providing us electrolyte in this study.

\section{Author contributions}

H.C. and L.C. conceived the ideas and designed the experiment. C.H. and H.C. carried out the materials preparation, characterization, and electrochemical testing. D.L. and Y.Z. did the XPS characterization. Y.S., A.-H.L., X.W., and W.L. provided valuable discussions.

C.H., H.C., and L.C. co-wrote the paper. All of the authors commented on the manuscript.

\section{Additional information}

Supplementary Information accompanies this paper at doi:10.1038/s41467-017-00656-8.

Competing interests: The authors declare no competing financial interests.

Reprints and permission information is available online at http://npg.nature.com/ reprintsandpermissions/

Publisher's note: Springer Nature remains neutral with regard to jurisdictional claims in published maps and institutional affiliations.

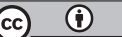

Open Access This article is licensed under a Creative Commons Attribution 4.0 International License, which permits use, sharing, adaptation, distribution and reproduction in any medium or format, as long as you give appropriate credit to the original author(s) and the source, provide a link to the Creative Commons license, and indicate if changes were made. The images or other third party material in this article are included in the article's Creative Commons license, unless indicated otherwise in a credit line to the material. If material is not included in the article's Creative Commons license and your intended use is not permitted by statutory regulation or exceeds the permitted use, you will need to obtain permission directly from the copyright holder. To view a copy of this license, visit http://creativecommons.org/ licenses/by/4.0/.

(C) The Author(s) 2017 